\title{
Explanation of Anomalous Behavior Observed in Impedance Eduction Techniques Using Measured Data
}

\author{
W. R. Watson* and M. G. Jones ${ }^{\dagger}$ \\ NASA Langley Research Center, Hampton, Virginia 23681-2199, USA
}

\begin{abstract}
Several enhancements that improve the accuracy and robustness of an impedance eduction technique that use an automatic optimizer are presented. These enhancements are then used to launch an intensive investigation into the cause of anomalous behavior that occurs for a small number of test conditions. This anomalous behavior is investigated for both a hardwall insert and a conventional liner. The primary conclusions of the study are that: (1) for the hard wall insert, the anomalies are due to narrow peaks in the objective function, (2) For the conventional liner, the anomalies are due to the presence of an extremely flat objective function, and (3) the anomalies appear to be triggered by inconsistencies between the duct propagation model and the measured data. At high frequencies, the duct propagation model may need to include the effects of higher-order duct modes, whereas at low frequencies, the effects of the mean boundary layer may have to be included.
\end{abstract}

\section{Nomenclature}

$[A],\{P\},\{F\} \quad=$ stiffness matrix, vector of unknown nodal coefficients, vector containing source effects

$c_{0}, M_{0}, \rho_{0} \quad=$ speed of sound, uniform flow Mach number, mean flow density

$d, f, k, t=$ cavity depth, frequency of sound source, freespace wavenumber $\left(\omega / c_{0}\right)$, time

$H, L, W \quad=$ height of duct, length of duct, width of duct

$i, \omega=$ unit imaginary number $(\sqrt{-1})$, circular frequency $(2 \pi f)$

$N, p \quad=$ number of lower wall microphones, acoustic pressure field

$L_{1}, L_{2} \quad=$ axial location of leading edge of test liner, axial location of trailing edge of test liner

$X, Y=$ unconstrained resistance variable, unconstrained reactance variable

$x, y, z=$ Cartesian coordinates

$\beta, \zeta=$ dimensionless admittance of test liner, dimensionless impedance of test liner

$\theta, \chi \quad=$ dimensionless resistance of test liner, dimensionless reactance of test liner

$\phi(\zeta),\|\| \quad=$ objective function, complex absolute value

Subscripts:

Meas, $s, t \quad=$ a measured quantity, a source plane quantity, an exit plane quantity

$\min , \max =$ minimum value of a quantity, maximum value of a quantity

\section{Introduction}

Increasingly stringent noise constraints in the United States and abroad have resulted in continued emphasis on the development of improved acoustic liner technologies to reduce the overall levels of fan noise radiated to communities surrounding airports. Consequently, various numerical methods have been developed by government, industry, and academia to support locally-reacting liner impedance evaluation. The numerical methods ${ }^{1-15}$ include codes based on modal and finite element analyses. Of these, finite element codes developed at the NASA Langley Research Center $(\mathrm{LaRC})^{4,5,11,12,14}$ have been demonstrated to provide quality results in ducts where a single plane wave mode can be isolated between one pair of opposite rigid walls. Further, over the last three decades, LaRC has developed a number of test rigs that obtain measured data for the evaluation of locally-reacting acoustic liner impedance in the presence

\footnotetext{
*Senior Research Scientist, Research Directorate, Computational AeroSciences Branch, Liner Physics Group, AIAA Associate Fellow.

${ }^{\dagger}$ Senior Research Scientist, Research Directorate, Structural Acoustics Branch, Head Liner Physics Group, AIAA Associate Fellow.
} 
of grazing flow. The most notable of these are the Grazing Incidence Tube ${ }^{16}$ (GIT) and the Curved Duct Test Rig (CDTR). ${ }^{17}$

In 2009, LaRC brought online a replacement flow duct for the GIT that is now referred to as the Grazing Flow Impedance Tube (GFIT). Lessons learned from the GIT were applied to the design of the GFIT. Some of the more interesting features of the new design are: (1) the use of a sealed test window to eliminate flow leakage effects experience in the GIT, (2) incorporation of a close-coupled, phased-array source section to provide increased sound source authority, (3) implementation of a modular design, so that portions of the duct can be easily interchanged (e.g., the source section and the test window can be alternated between inlet and aft-duct configurations), (4) implementation of a dual-axis traverse probe system for efficient flow profile measurement, and (5) implementation of a rectangular cross-section so that high-order horizontal and vertical modes cut on at different frequencies.

Impedances are educed for test liners in the GFIT using an impedance eduction procedure that has been well tested in the GIT. ${ }^{4}$ Although the method works well in approximately $90 \%$ of the cases, some anomalies have arisen. These anomalies are characterized by precipitous rises (or drops) in the educed impedance in the low or high frequency ends of the impedance spectrum. Because these educed impedance values are out of line with the educed impedance at surrounding frequencies in the spectrum, they are said to be anomalous. To date, these anomalies have been observed when the impedance eduction technique is implemented using measured data. The primary objective of this paper is to investigate the root cause of these anomalies. A secondary objective of this paper is to describe several enhancements that were made to the original impedance eduction procedure ${ }^{4}$ in order to improve its accuracy and robustness.

\section{Governing Equations and Boundary Conditions}

A schematic of the GFIT is given in Fig. 1. An orthogonal coordinate system is used in which $z$ is the axial coordinate and the two transverse coordinate are denoted by $x$ and $y$. The source and termination planes of the duct are located at $z=0$ and $z=L$, respectively. The upper wall contains the test liner which is $\left(L_{2}-L_{1}\right)$ units in length and the remainder of the duct is rigid. The leading and trailing edges of the liner are located at $z=L_{1}$, and $z=L_{2}$, respectively. Note that the lower wall of the duct contains a number of flush-mounted microphones in the liner test section. Acoustic pressure data from these flush-mounted microphones are used to educe the impedance. Microphones are also

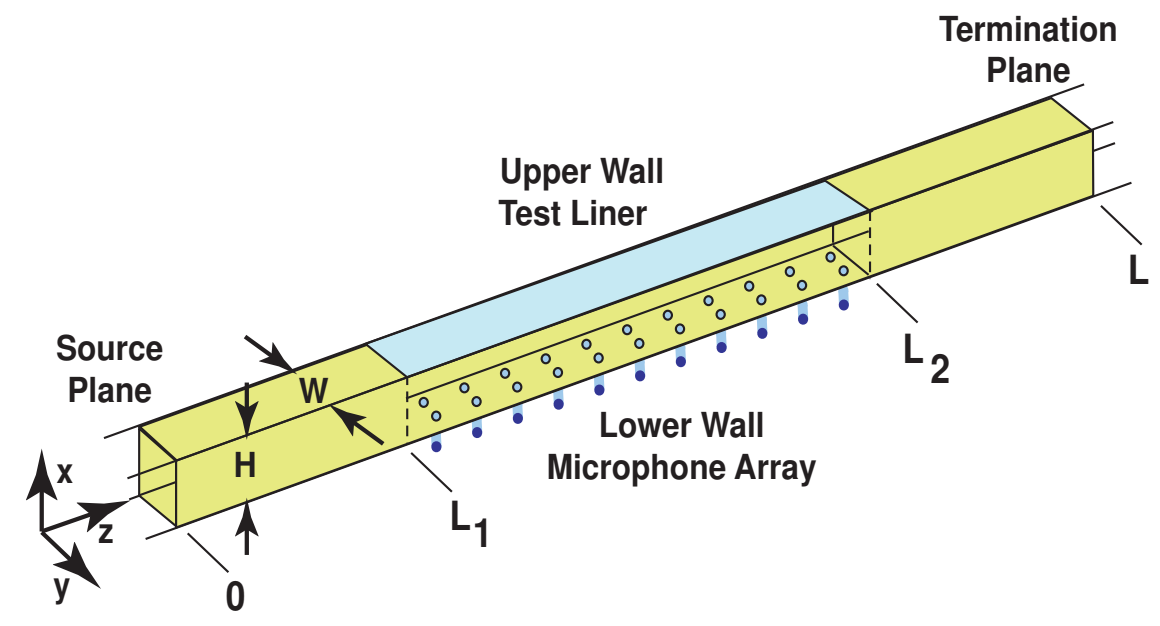

Figure 1. Schematic of LaRC Grazing Flow Impedance Tube.

embedded in the rigid wall portion of duct upstream and downstream of the test liner. Although these microphones are not shown in Fig. 1, they are used to measure the source and exit plane boundary conditions. Assuming that the source frequency is sufficiently low so that only planar acoustic waves are supported in the horizontal direction (i.e., the $y$ direction) and that the mean flow field is constant, the acoustic pressure field in the duct depicted in Fig. 1 can be shown to satisfy the 2D convected Helmholtz equation ${ }^{18}$

$$
\left(1-M_{0}^{2}\right) \frac{\partial^{2} p(z, x)}{\partial z^{2}}+\frac{\partial^{2} p(z, x)}{\partial x^{2}}-2 i k M_{0} \frac{\partial p(z, x)}{\partial z}+k^{2} p(z, x)=0
$$


where a time-harmonic dependence of the form $e^{i \omega t}$ has been assumed. Readers are reminded that the assumption of a uniform mean flow field may be difficult to achieve at the higher flow speeds inside of a duct with small cross-sectional dimensions such as the GFIT. The convected Helmholtz equation is solved subject to the source plane, termination plane, and lower wall boundary conditions

$$
p(0, x)=p_{s}, \quad p(L, x)=p_{t}, \quad \frac{\partial p(z, 0)}{\partial x}=0
$$

Flush-mounted wall microphones located at the source and exit planes are used to measure $p_{s}$ and $p_{t}$, respectively. The upper wall impedance boundary condition has been derived and discussed in detail by Myers. ${ }^{19}$ When written in terms of the acoustic pressure this boundary condition is:

$$
-\frac{\partial p(z, H)}{\partial x}=i k\left[\frac{p(z, H)}{\zeta}\right]+2 M_{0} \frac{\partial}{\partial z}\left[\frac{p(z, H)}{\zeta}\right]+\frac{M_{0}^{2}}{i k} \frac{\partial^{2}}{\partial z^{2}}\left[\frac{p(z, H)}{\zeta}\right]
$$

In Eq. (3), $\zeta=\theta+i \chi$ is the unknown dimensionless impedance of the test liner and its reciprocal, $\beta=1 / \zeta$, is taken as zero along the rigid portion of the upper wall. The parameters $\theta$ and $\chi$ are the unknown dimensionless resistance and reactance, respectively, of the test liner. Throughout this paper, all resistances and reactances are made dimensionless by dividing by the characteristic impedance, $\rho_{0} c_{0}$, of an outgoing plane wave in the duct and the International system of units are used. Note that for a specified wall impedance, $\zeta$, Eqs. (1)-(3) constitute a boundary value problem $(\mathrm{BVP})^{18}$ that can be solved numerically to determine a unique set of lower wall acoustic pressures, $p\left(z_{I}, 0\right)$, at the flush-mounted microphone locations located at $z=z_{I}$. This uniqueness of the lower wall acoustic pressure profile satisfying the solution to the $\mathrm{BVP}^{18}$ provides the basis for the impedance eduction technique investigated in this paper.

\section{Impedance Eduction Technique}

The technique used to educe the impedance parallels that used in an earlier work ${ }^{4}$ and only a brief summary of the technique is provided in this paper. The impedance eduction method is summarized as follows:

1. Measure the source-plane acoustic pressure, $p_{\mathrm{s}}$, and termination-plane acoustic pressure, $p_{\mathrm{t}}$, each of which is assumed to be planar.

2. Measure the set of acoustic wall pressures, $p_{\text {Meas }}\left(z_{I}\right)$, along the array of flush-mounted wall microphones along the lower wall opposite the test liner.

3. Using an initial estimate for the test liner impedance, $\zeta$, solve the BVP defined by Eqs. (1)-(3) to obtain a unique set of lower wall acoustic pressures.

4. Compare the measured set of lower wall acoustic pressures to those obtained from the solution to the BVP. If these two sets of lower acoustic wall pressures match (to within a predetermined tolerance), then the initial guess for the test liner impedance is the impedance of the test liner.

5. If the two sets of lower wall acoustic pressures do not match, then the unknown impedance, $\zeta$, of the test liner is iterated upon (via an automated search algorithm) until the two sets of lower wall acoustic pressures match (to within the predetermined tolerance). The resulting impedance is the unknown impedance of the test liner.

It is convenient to recast the above impedance eduction procedure as an optimization problem. This is achieved by finding the impedance that maximizes the objective function, $\phi(\zeta)$

$$
\phi(\zeta)=\frac{1}{\sqrt{\sum_{I=1}^{I=N}\left\|p_{\text {Meas }}\left(z_{I}\right)-p\left(z_{I}, 0\right)\right\|}}
$$

In the current implementation of the optimization method, the numerical solution to the BVP is obtained using a cubic finite element methodology (FEM). Thus, the the solution for the acoustic pressure field, $p$, is represented as a polynomial that is the product of cubic 2-D Hermite polynomials and unknown nodal coefficients, $p_{I}$. The unknown nodal coefficients, $p_{I}$, are shown to satisfy the large but sparse matrix equation

$$
[A]\{P\}=\{F\}
$$


Here the solution vector $\{P\}$ provides the values of the unknown nodal coefficients. The solution for the nodal coefficients are then used to determine the unique set of lower wall acoustic pressures, $p\left(z_{I}, 0\right)$, for constructing the objective function, $\phi$. Further details of the finite element analysis are provide in a previous paper. ${ }^{4}$ The optimization algorithm chosen to maximize the objective function is Stewart's adaptation of the Davidon-Fletcher-Powell (SDFP) optimization algorithm. ${ }^{20}$ This is a gradient-based optimizer that uses a finite difference approximation to obtain the gradient of the objective function and is chosen because of its computational efficiency.

\section{Enhancements to the Impedance Eduction Method}

Several enhancements to the impedance eduction method used in Ref. 4 have been implemented in this paper. These enhancements are described in this section.

\section{A. Accuracy Improvements}

In this paper, the accuracy of the computational model is increased by switching from an exit impedance boundary condition at $z=L$, to an exit pressure boundary condition. One lesson learned in a previous study ${ }^{14}$ is that the accuracy of the computational model is increased by switching from an exit impedance boundary condition at $z=L$, to the exit pressure boundary condition defined by Eq. (2). This increase in accuracy of the computational model occurs because a direct measurement of the exit pressure, $p_{s}$, is available to implement into the exit pressure boundary condition (see Eq. (2)). However, the exit impedance needed for the exit impedance boundary condition is not directly measurable. Consequently, the exit impedance must be approximated from the measured lower wall pressures. This leads to some loss of accuracy in the computational model. The accuracy of the solution vector, $\{P\}$, from Eq. (5) is also improved by switching from the $\mathrm{SGI}^{21}$ sparse equation solver, ZPSLDU, to the Intel ${ }^{22}$ sparse equation solver, PARDISO, and then performing iterative refinement. The SGI solver, ZPSLDU, that was used in earlier works does not provide the capability to improve the solution vector by iterative refinement.

\section{B. Use of a Constrained Optimization}

The SDFP algorithm is defined only for unconstrained optimization problems. Subsequently, the educed resistance can sometimes converge to a negative value. A negative resistance corresponds to an active source of sound energy other than that at the source plane or to a background noise level that is higher than the sound source. The capability to constrain both the dimensionless resistance and reactance has been implemented into the current method. The constraints are implemented as described in the following paragraph.

Let the unconstrained design variables used by SDFP be denoted by $X$ and $Y$, and let the constrained resistance and reactance of the test liner be defined as:

$$
\begin{aligned}
& \theta=\theta_{\text {min }}+\left(\theta_{\text {max }}-\theta_{\text {min }}\right) \sin ^{2}(X) \\
& \chi=\chi_{\text {min }}+\left(\chi_{\text {max }}-\chi_{\text {min }}\right) \sin ^{2}(Y)
\end{aligned}
$$

where $\left(\theta_{\min }, \theta_{\max }\right)$ and $\left(\chi_{\min }, \chi_{\max }\right)$ are the minimum and maximum values, respectively, of the dimensionless resistance, $\theta$, and reactance, $\chi$. The unconstrained optimization algorithm (SDFP) is now run with $X$ and $Y$ as the unconstrained design variables. In the current implementation, the minimum and maximum values are set as follows:

$$
\begin{gathered}
\theta_{\max }=\chi_{\max }=10 \\
\theta_{\min }=0, \quad \chi_{\min }=-10
\end{gathered}
$$

These ranges are expected to cover the majority of the conventional liners to be tested in the GFIT.

\section{Use of Multiple Starting Locations}

Because local optimizers such as SDFP can become stuck in regions of the impedance plane where the objective function is flat, overshoot the optimum on a sharp and peaky surface, or converge to local optima, the following strategy is used to increase the likelihood that a global optimum is acheived

1. The constrained design space is divided into four quadrants (quadrant I, quadrant II, quadrant III, and quadrant IV) that are determined as follows: 
(a) For quadrant I: $\theta_{\min } \leq \theta \leq \frac{1}{2}\left(\theta_{\max }+\theta_{\min }\right)$, and $\chi_{\min } \leq \chi \leq \frac{1}{2}\left(\chi_{\max }+\chi_{\min }\right)$

(b) For quadrant II: $\frac{1}{2}\left(\theta_{\max }+\theta_{\min }\right) \leq \theta \leq \theta_{\max }$, and $\chi_{\min } \leq \chi \leq \frac{1}{2}\left(\chi_{\max }+\chi_{\min }\right)$

(c) For quadrant III: $\frac{1}{2}\left(\theta_{\max }+\theta_{\min }\right) \leq \theta \leq \theta_{\max }$, and $\frac{1}{2}\left(\chi_{\max }+\chi_{\min }\right) \leq \chi \leq \chi_{\max }$

(d) For quadrant IV: $\theta_{\min } \leq \theta \leq \frac{1}{2}\left(\theta_{\max }+\theta_{\min }\right)$, and $\frac{1}{2}\left(\chi_{\max }+\chi_{\min }\right) \leq \chi \leq \chi_{\max }$

2. SDFP is run four times using the midpoint of each quadrant as the starting location (note: these four starting locations are used whether SDFP is run constrained or unconstrained)

3. If the impedances obtained from each of the four starting locations are identical, then this impedance is taken as the impedance of the test liner

4. If the impedances obtained from each of the four runs are not identical, then the impedance with the largest objective function is taken as the impedance of the test liner. The rationale for this decision is that the impedance with the largest objective function is the one that best matches the experimental data (see Eqs. (4)).

\section{Results and Discussion}

An in-house computer code that implements the enhancements to the impedance eduction technique has been developed. The code uses Fortran 90 with double precision (i.e., 64 bit) arithmetic and was designed to run in a shared memory environment. This approach has been chosen because the primary hardware to be utilized is a shared memory system. In the current implementation, the resistance, $\theta$, and reactance, $\chi$, of the test liner are the design variables for the unconstrained optimization while the unconstrained variables $X$ and $Y$ are used as design variables for the constrained optimization runs. For each type of optimization (constrained or unconstrained) the four unique starting values discussed in the previous subsection are used.

The computational cost of the impedance eduction grows as the FEM spatial grid increases in density. Prior to impedance eductions, a grid refinement study was conducted at each frequency of interest to determine the coarsest grid for accurate impedance eductions. This grid refinement study is not presented for the sake of brevity. However, four separate finite element grids (each obtained from doubling of a coarser grid) were investigated. The FEM grid used in the results to follow was a 161 x 26 evenly spaced grid, where the largest number of points (i.e., 161) was along the axis of the duct. This was the second coarsest grid used in the grid refinement study for which the educed impedance was observed to have converged.

Several examples are now presented to determine the root cause of the anomalies encountered in hard and soft wall ducts. Data for the impedance eduction is obtained using the GFIT for which $L_{1}=203 \mathrm{~mm}, L_{2}=610 \mathrm{~mm}, L=1061$ $\mathrm{mm}, W=50.8 \mathrm{~mm}$, and $H=63.5 \mathrm{~mm}$ (see Fig. 1). Source plane, termination plane, and lower wall acoustic pressure measurements, together with mean flow data are acquired at three average centerline Mach numbers $(0.0,0.3$, and 0.5 ), for an incident sound pressure level of $130 \mathrm{~dB}$, and for source frequencies ranging from 0.4 to $3.0 \mathrm{kHz}$ in increments of $0.2 \mathrm{kHz}$. A total of 53 flush mounted microphones were imbedded into the lower wall of the GFIT to obtain a measurement of the lower wall acoustic pressures, $p_{\text {Meas }}\left(z_{I}\right)$, for the impedance eductions. Acoustic pressure measurements taken at the first and last microphones are used as the source and termination plane acoustic pressure. Plane waves are assumed, so $p_{s}$ and $p_{t}$ are assumed independent of the transverse coordinate, $x$.

Results are presented for both a hard wall insert and a test liner. The hard wall insert is a $12.7 \mathrm{~mm}$ thick stainless steel plate and is chosen because it provides a liner for which the dimensionless admittance, $\beta$, is known. The soft test liner is a single-layer, conventional, perforate-over-honeycomb liner. This liner was chosen because it is representative of liners commonly used for the reduction of fan noise in current aircraft engine nacelles. The facesheet for this liner has $0.991 \mathrm{~mm}$-diameter holes, an open area ratio of 0.087 , and a thickness of $0.635 \mathrm{~mm}$. The depth of the cavity is $38.18 \mathrm{~mm}$. The optimization code was run both constrained and unconstrained. The constrained and the unconstrained optimization codes were observed to return nearly identical educed impedances except for test conditions where anomalies were observed to occur. Often, the unconstrained optimization code returned a negative resistance at test conditions where an anomaly occurred. Therefore unless stated otherwise, the results reported in this section were obtained using the constrained optimization code.

\section{A. Educed admittance of the Hard Wall Insert}

Calculations in this subsection are presented to demonstrate how the impedance eduction technique performs using the hard wall insert. It is noted that for a hard wall, the resistance and reactance are extremely large. Therefore, in 
the simulations to follow, we present the educed dimensionless conductance $(\operatorname{Real}(\beta))$ and the educed dimensionless susceptance (Imaginary $(\beta))$ for the hard wall insert. The dimensionless conductance and susceptance of the hard wall insert are expected to be extremely small (i.e., identically zero for a perfectly rigid insert).

Figure 2 shows the educed dimensionless conductance of the hard wall insert at the three average centerline Mach numbers. Note that the impedance eduction code returns nearly a zero conductance (as expected) for each test condition (i.e, Mach number and frequency). Figure 3 shows the educed susceptance for the hard wall insert. The educed susceptance is also observed to be in good agreement with the expected value of zero, except for four test conditions where anomalies are observed to occur. These four test conditions are located at $0.6 \mathrm{kHz}$ for Mach $0.0,1.2 \mathrm{kHz}$ for Mach 0.3, and 1.2 and $1.8 \mathrm{kHz}$ for Mach 0.5. It is reemphasized that the constrained and unconstrained version of the code were observed to returned different susceptance values at each of these four test conditions.

To determine the root cause of the anomalies for the hard wall insert, a detailed contour plot of the objective function was constructed at each of the four test conditions where anomalies were observed to occur. The contour plot data was then thoroughly examined to determine the source of any anomaly. Figure 4 is a contour plot for the test condition corresponding to Mach 0.0 and $0.6 \mathrm{kHz}$. This contour plot is typical of what was obtained at the other three test conditions where anomalies occur. Here, the brighter colors (i.e., the red region) corresponds to regions where the objective function is the highest and the darker colors (i.e., the blue regions) corresponds to regions where the value of the objective function is low. An area corresponding to a large value of the objective function is in a region of the complex impedance plane where the match between the measured acoustic pressure and that computed by the FEM are in good agreement. The impedance of the test sample lies is the red region of the contour plot. Note that the eye (i.e., the red region) of the contour plot has a small diameter of less that 0.05 dimensionless units and the objective function drops sharply in value at surrounding locations in the conductance/susceptance space. Also, the dimensionless susceptance is observed to have a value close to zero (as expected) from the contour plot. However, the optimizer returned a value of approximately 3.6 (see Fig. 3). Thus, the root cause of the anomaly was determined to be that the gradient-based optimizer was either overshooting or undershooting the correct optimum point because of an extremely sharp gradient (i.e., narrow peak in the objective function). Thus the switch from a gradient-base to a nongradient base optimizer may be necessary to minimize this effect. Further, there is no anomaly in the high frequency end of the spectra for the hard wall insert. This appears due to the fact that there are not significant axial discontinuities in the hard wall duct to scatter acoustic energy into higher-order transverse modes. It will be shown later that high frequency anomalies do occur when a liner is inserted and this may be due to axial impedance discontinuities which are present at the leading and trailing edges of the liner.

\section{B. Educed Impedance of Conventional Liner}

The educed dimensionless resistance of the conventional liner for each of the three uniform flow Mach numbers is given in Fig 5. Note that the resistance of the conventional liner is nearly independent of frequency for each of the three uniform flow Mach numbers. At Mach 0.0, the conventional liner is a low resistance liner. However, the resistance increases with the mean flow Mach number. This resistance spectra is typical of what is expected for a conventional liner with two exceptions. First, the precipitous drop in resistance for Mach 0.5 and increase in the resistance at Mach 0.3 at a frequency of $0.4 \mathrm{kHz}$ are out of line with their values at $0.6 \mathrm{kHz}$. Second, there is considerable oscillation in the resistance spectra at frequencies beyond $2.7 \mathrm{kHz}$. These oscillations are inconsistent with the resistance spectra below $2.7 \mathrm{kHz}$ for which little oscillation occur. Thus, the educed resistance for Mach 0.5 and $0.3 \mathrm{at} 0.4 \mathrm{kHz}$ and at frequencies beyond $2.7 \mathrm{kHz}$ are therefore said to be anomalous (the impedance spectrum is generally expected to be a relatively smooth function of frequency, except for frequencies near anti-resonance). Further, for these two exceptions, the constrained and unconstrained optimizations did not educe the same dimensionless resistance and the educed resistance was sensitive to the initial guess provided to SDFP. It is worth noting also that for Mach 0.0, anomalies occur only for frequencies above $2.7 \mathrm{kHz}$ and the educed resistance at all frequencies was independent of the initial guess provided to SDFP. Further, the cut on frequency for higher-order duct mode propagation in the GFIT is approximately $2.7 \mathrm{kHz}$.

The educed reactance spectra for the conventional liner is shown in Fig. 6. The educed reactance spectrum at each Mach number is expected to have a $-\cot (k d)$ behavior. Note that the dimensionless reactance has the expected trend except in that portion of the spectra where anomalies have already been identified in the resistance spectra (i.e., at frequencies beyond $2.7 \mathrm{kHz}$ and at the low frequency end of the spectra). Also, the educed reactance at Mach 0.0 was also observed to be insensitive to the initial guess provide to SDFP (even at frequencies where anomalies occur).

Figures 7 shows a contour plot of the objective function at the low frequency end of the spectrum where there is an anomaly in the educed reactance (i.e., at Mach 0.3 and $0.4 \mathrm{kHz}$ ). This contour plot is typical of those obtained in the low frequency end of the spectrum when an anomaly occurs. Note that there is a primary eye and secondary 
eyes in the objective function. The primary eye of the contour (the one containing the largest value of the objective function) is quite diffused at this frequency. This indicates a large amount of uncertainty in the educed impedance. The diffused primary eye also implies a relatively flat objective function in the vicinity of the optimum point. Obtaining the optimum on a relatively flat surface is a major challenge for a gradient based optimizer such as SDFP. Other classes of optimizers that require only a function evaluation may prove more successful on these flat surfaces. It should be noted that the eye of the contour plot at frequencies where anomalies did not occur was nearly an order of magnitude smaller in diameter than that of the primary eye in Fig. 7 and the secondary eyes depicted in Fig. 7 did not occur.

Figure 8 is a contour plot of the objective function, $\phi(\zeta)$, at a Mach number of zero $\left(M_{0}=0.0\right)$ and frequency of $3.0 \mathrm{kHz}$ where an anomaly occurs in the educed resistance. The characteristics of this contour plot are typical of those obtained for frequencies beyond the cut on frequency of higher-order duct modes (i.e., $f \geq 2.7 \mathrm{kHz}$ ) where anomalies occur. Observe that the primary eye of the contour (i.e., the one containing the largest value of the objective function) is extremely large and non-physical and the contour plot also contains secondary eyes in the contour plot. This occurs because there may be a breakdown in the assumptions used in the current analysis. This breakdown can occur for two reasons. First, higher-order duct modes are generated in the vicinity of the leading and trailing edge of the liner. These higher-order duct modes will propagate with little decay near their cut on frequency and can carry acoustic energy to the source and duct termination planes. The effects of these higher-order duct modes (at the source and termination planes) have been neglected in the current analysis. Second, at frequencies above the cut on frequency of higher-order modes, the sound source may not contain just planar waves, whereas only plane waves are assumed for the sources used in this study. It should also be noted that the diameter of the eye of the contours grow with increasing Mach number at frequencies beyond the cut on frequency of higher-order duct modes.

The most plausible explanation for the anomalies experienced in these results is an inconsistency between the assumptions used in the FEM model and the measured data. For example, the FEM analysis assumes that higherorder duct modes do not contain acoustic energy at the source and termination planes. This assumption is unlikely to be supported by the measured data at frequencies above the cut on frequency of higher-order duct modes. It was noted earlier that the cut on frequency for higher-order duct modes in the GFIT is approximately $2.7 \mathrm{kHz}$. Therefore, the anomaly observed for frequencies beyond $2.7 \mathrm{kHz}$ are likely due to the neglect of higher-order duct modes at the source and termination planes of the GFIT. Although the current FEM analysis can account for the effects of these higher-order modes, transversing probes were not available in the GFIT to measure the acoustic energy in these modes. Further, the low frequency anomaly at $0.4 \mathrm{kHz}$ is likely due to neglecting the mean boundary layer in the FEM analysis (the current FEM analysis assumes uniform flow). A back of the envelope calculation shows that a duct with small cross-sectional dimensions such as the GFIT, has a boundary layer covering the full cross-section of duct. In this situation the convected Helmholtz Equation (see Eq. (1)) should be replaced by the linearized Euler Equations as discussed in Ref. 11.

\section{Conclusions}

Based upon the results of this study, the following conclusions can be drawn:

1. Eduction anomalies occur for a relatively small number of test conditions (i.e., Mach numbers and frequencies)

2. For the hard wall insert, the anomalies are due to a sharp (narrow peak) objective function

3. For the conventional liner, the anomalies are due to the presence of an extremely flat objective function. The flatness of the objective function is an increasing function of the mean flow Mach number. In the absence of mean flow, the conventional liner anomalies occur only at frequencies above the cut on frequency of higherorder duct modes, and they are insensitive to the initial guess provided to the optimization algorithm. When mean flow is present, the anomalies occur at both the low and high frequency end of the spectrum, and the location in the impedance plane where the anomalies occur is quite sensitive to the initial guess provided to the optimization algorithm

4. All indications from this research suggest that the anomalies are triggered by an inconsistency between the duct propagation model and the measured data. At high frequencies, the duct propagation model may need to include the effects of higher-order modes, whereas at low frequencies, the effects of the mean boundary layer may have to be included. 


\section{Acknowledgement}

This work was supported by the Subsonic Fixed Wing Project of NASA's Fundamental Aeronautics Program.

\section{References}

\footnotetext{
${ }^{1}$ Armstrong, D. L., Beckemeyer, R. J., and Olsen, R. F., “Impedance Measurements of Acoustic Duct Liners With Grazing Flow,” Paper presented at the 87th Meeting of the Acoustical Society of America, New York, NY, 1974.

${ }^{2}$ Watson W. R., "A New Method for Determining Acoustic-Liner Admittance in Ducts with Sheared Flow in Two Cross-Sectional Directions," NASA TP-2518, 1985.

${ }^{3}$ Watson, W. R., Tanner, S. E., and Parrott, T. L., "Optimization Method for Educing Variable-Impedance Liner Properties," AIAA Journal, Vol. 36, No. 1, 1998, pp. 18-23.

${ }^{4}$ Watson, W. R., Jones, M. G., and Parrott, T. L., "Validation of an Impedance Eduction Method in Flow," AIAA Journal, Vol. 37, No. 7, July 1999, pp. 818-824.

${ }^{5}$ Watson, W. R., Tracy, M. B., Jones, M. G., and Parrott, T. L.,"Impedance Eduction in the Presence of Shear Flow," AIAA paper 2001-2236, May 2001.

${ }^{6}$ Jones, M. G., Parrott, T. L., and Watson, W. R., "Comparison of Acoustic Impedance Eduction Techniques for Locally-Reacting Liners," AIAA Paper 2003-3306, May 2003.

${ }^{7}$ Elnady, T. and Boden, H., "An Inverse Analytical Method for Extracting Liner Impedance from Pressure Measurements " AIAA Paper 2004-2836, May 2004.

${ }^{8}$ Jones, M. G., Watson, W. R., Tracy, M. B., and Parrott, T. L., "Comparison of Two Waveguide Methods for Educing Liner Impedance in Grazing Flow," AIAA Journal, Vol. 42, No. 2, 2004, pp. 232-240.

${ }^{9}$ Auregan, Y., Leroux, M., and Pagneux, V., "Measurement of Liner Impedance With Flow by an Inverse Method," AIAA Paper 2004-2838, May 2004.

${ }^{10}$ Watson, W. R., Jones, M. G., and Parrott, T. L., “A Quasi-3-D Theory for Impedance Eduction in Uniform Grazing Flow,” AIAA Paper 2005-2848, May 2005.

${ }^{11}$ Watson, W. R., Jones, M. G., and Parrott, T. L., "Comparison of a Convected Helmholtz and Euler Model for Impedance Eduction in Flow," AIAA Paper 2006-2643, May 2006.

${ }^{12}$ Watson, W. R. and Jones, M. G, "Impedance Eduction in Ducts with Higher Order Modes and Flow," AIAA Paper 2009-3236, May 2009.

${ }^{13}$ Elnady, T., Musharraf, M., Boden, H., and Elhadidi, B., "Validation of an Inverse Analytical Technique to Educe Liner Impedance with Grazing Flow," AIAA Paper 2006-2639, May 2006

${ }^{14}$ Watson, W., Jones, M., and Parrott, T., “ Investigation of an Anomaly Observed in Impedance Eduction Techniques," AIAA Paper 2008-3013, May 2008

${ }^{15}$ Jing, X., Peng, S., and Sun, X., "A Straightforward Method for Wall Impedance Eduction in a Flow Duct," J. Acoust. Soc. Am., Vol. 124, No. 1, July 2008.

${ }^{16}$ Jones, M. G., Watson, W. R., Parrott, T. L., and Smith, C. D., "Design and Evaluation of Modifications to the NASA Langley Flow Impedance Tube," AIAA Paper 2004-2837, May 2004.

${ }^{17}$ Gerhold, C., Cabell, R., and Brown, M., "Development of an Experimental Rig for Investigation of Higher Order Modes in Ducts," AIAA Paper 2006-2637, May 2006.

${ }^{18}$ Kraft, R. E., "Theory and Measurement of Acoustic Wave Propagation in Multi-Sectioned Rectangular Ducts," Ph.D. dissertation, Department of Aerospace Engineering, University of Cincinnati, Cincinnati Ohio, 1976, pp. 78-85.

${ }^{19}$ Myers, M. K., "On the Acoustic Boundary Condition in the Presence of Flow," Journal of Sound and Vibration, Vol. 71, No. 3, 1980, pp.429-434.

${ }^{20}$ Stewart, G. W. III, “A Modification of Davidon's Minimization Method to Accept Difference Approximations of Derivatives," Journal of ACM, Vol. 14, No. 1, 1967, pp. 72-83.

${ }^{21}$ SGI Inc., "Scientific Computing Software Library (SCSL) Users Guide," Document Number:007-4325-001, December 30, 2002. [http://www.uni-kiel.de/rz/altix-doc/doc/sgi-propack-3/SCSL_UG/sgi_html/ch03.html\#id5189104. Accessed 5/15/2010.]

${ }^{22}$ Intel Inc., "Intel Math Kernal Library for Linux* OS Users Guide," Document Number: 314774-007US," August 2008. [http://developer.intel.com. Accessed 5/15/2010.]
} 


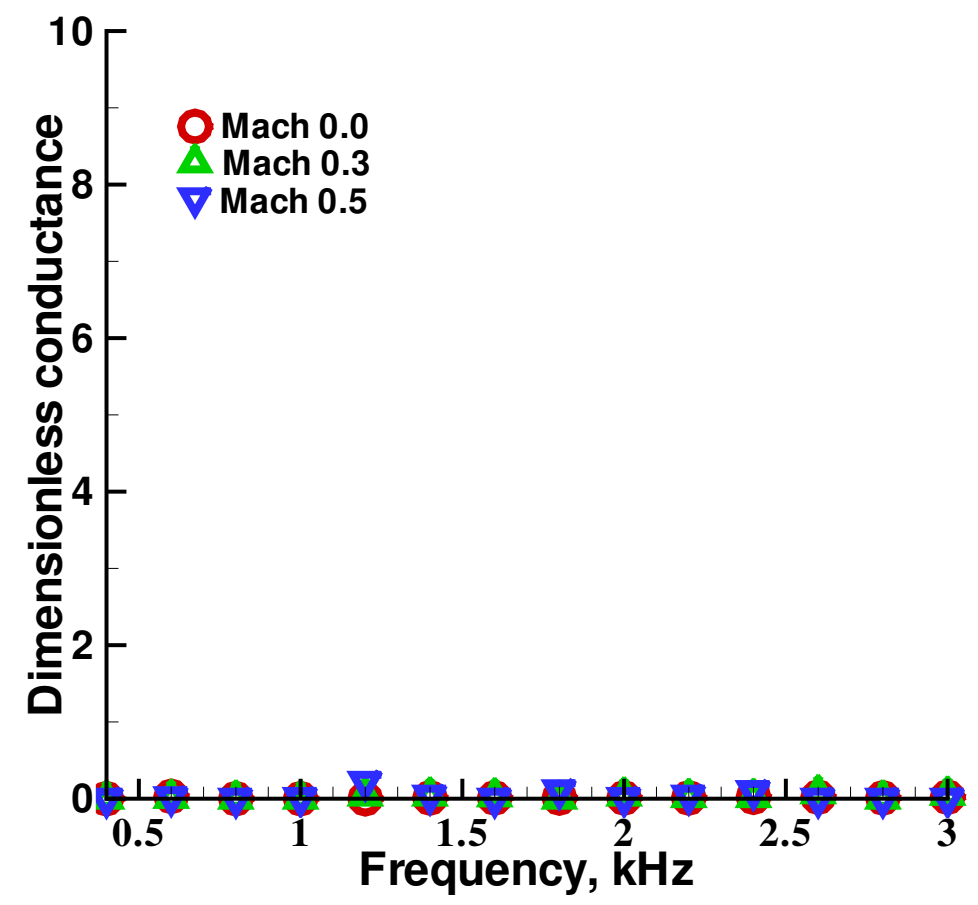

Figure 2. Educed dimensionless conductance for the hard wall insert.

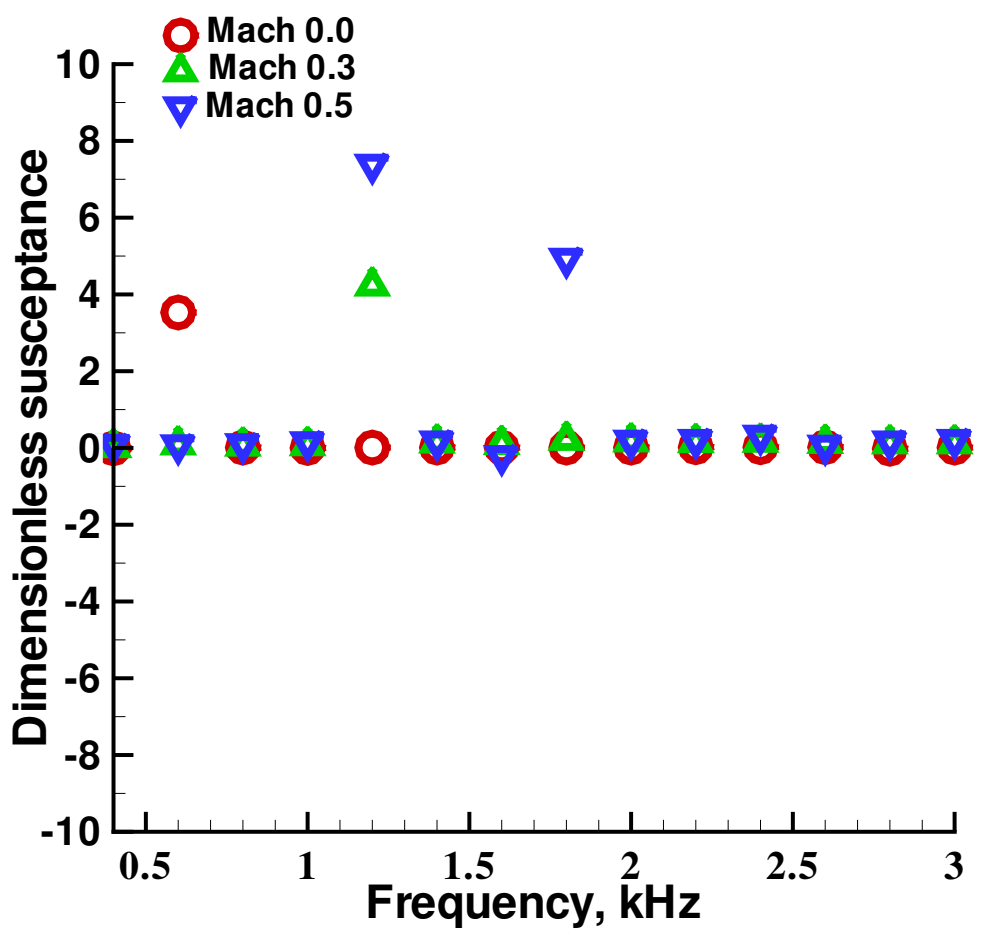

Figure 3. Educed dimensionless susceptance for the hard wall insert. 


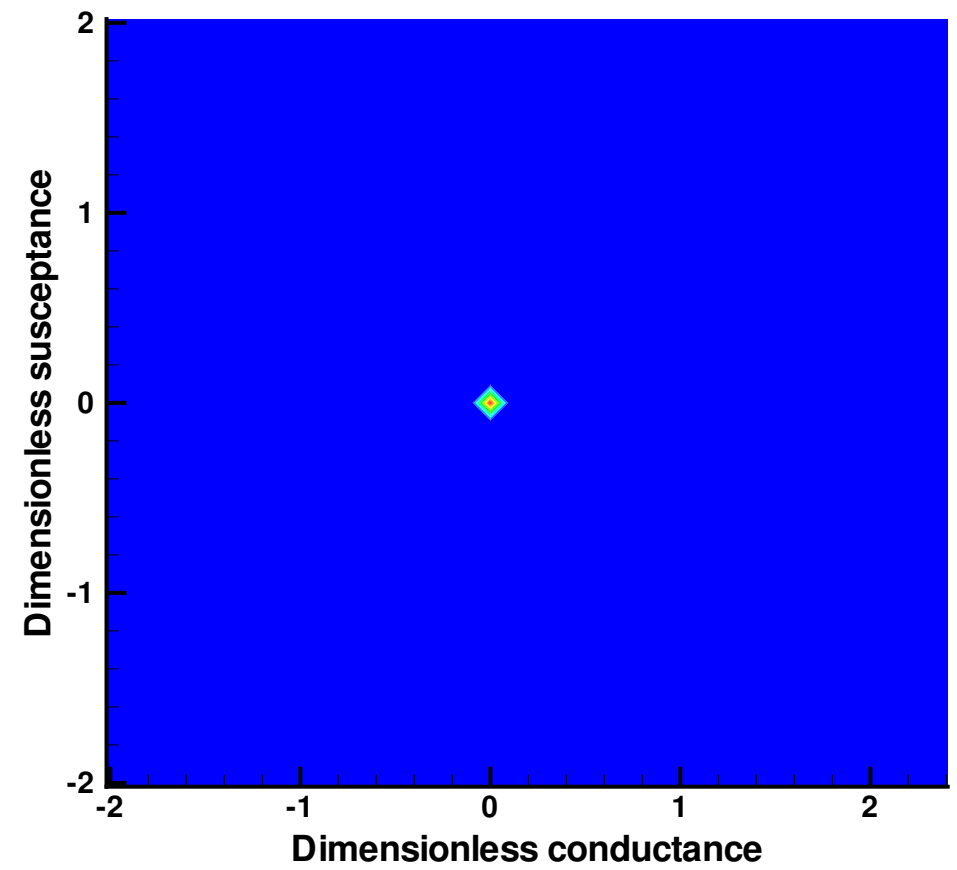

Figure 4. Contour plot of objective function for hard wall insert at Mach 0.0 and $0.6 \mathrm{kHz}$.

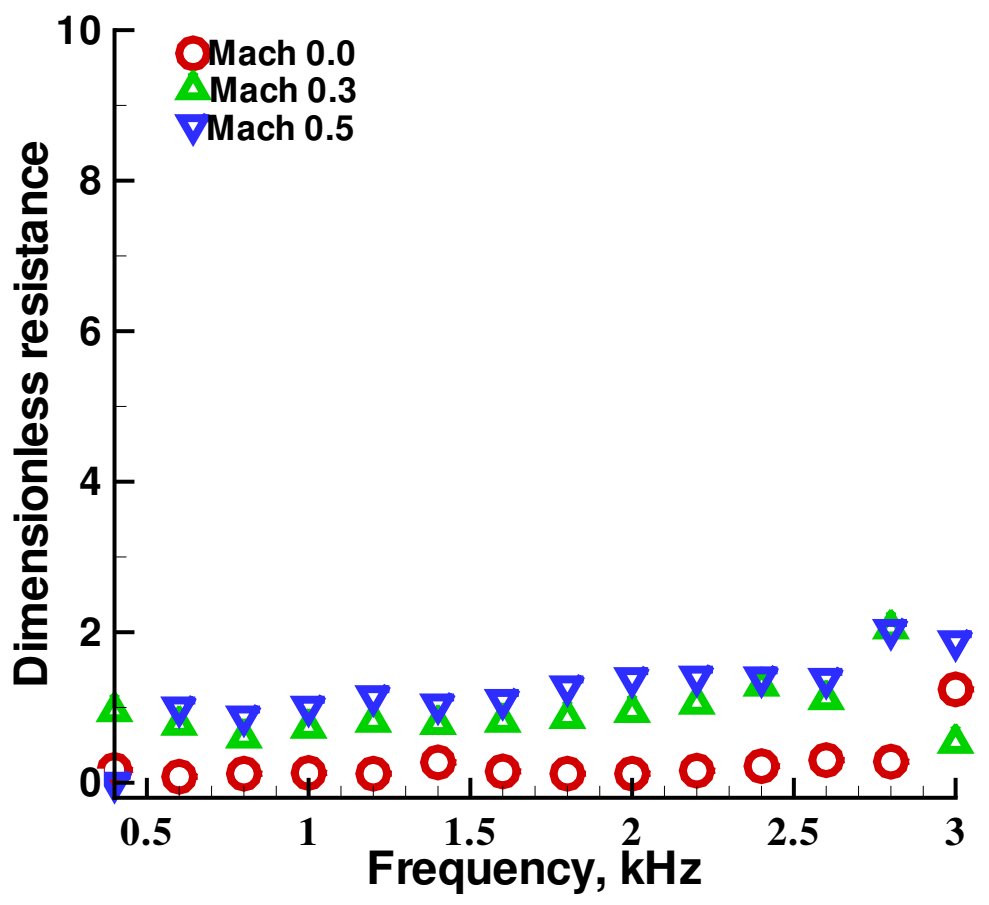

Figure 5. Educed dimensionless resistance for the conventional liner. 


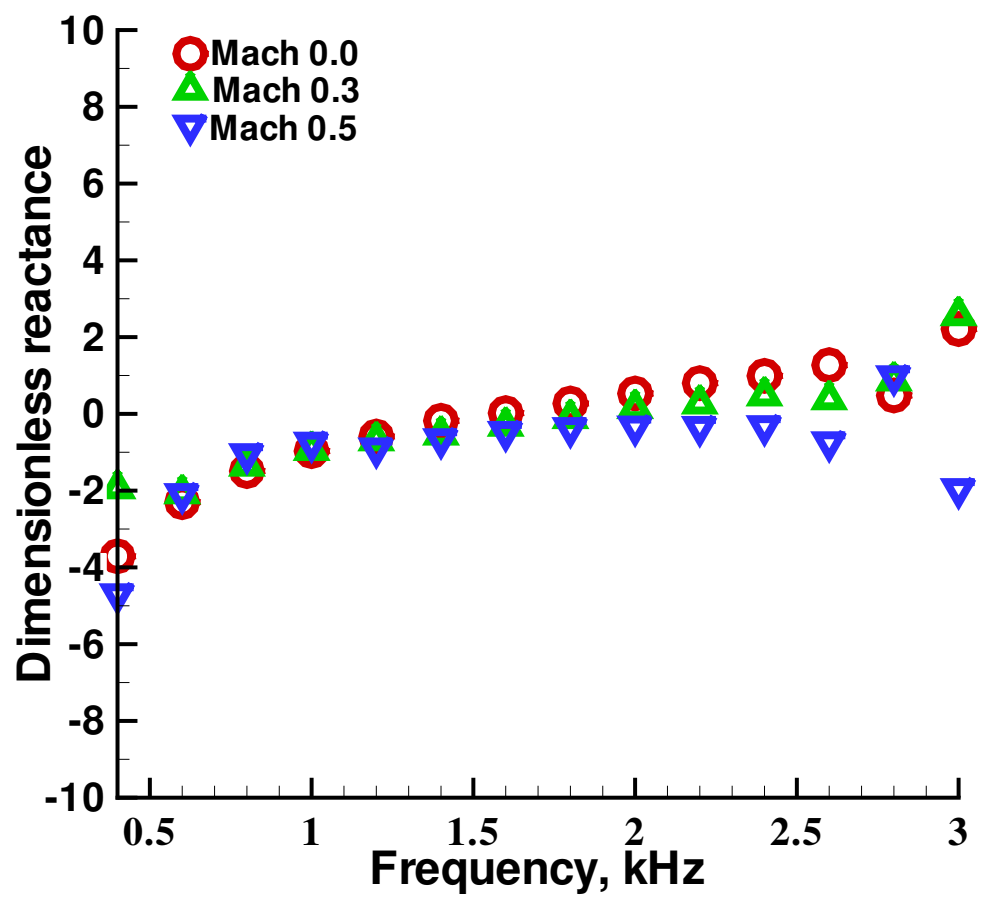

Figure 6. Educed dimensionless reactance for the conventional liner.

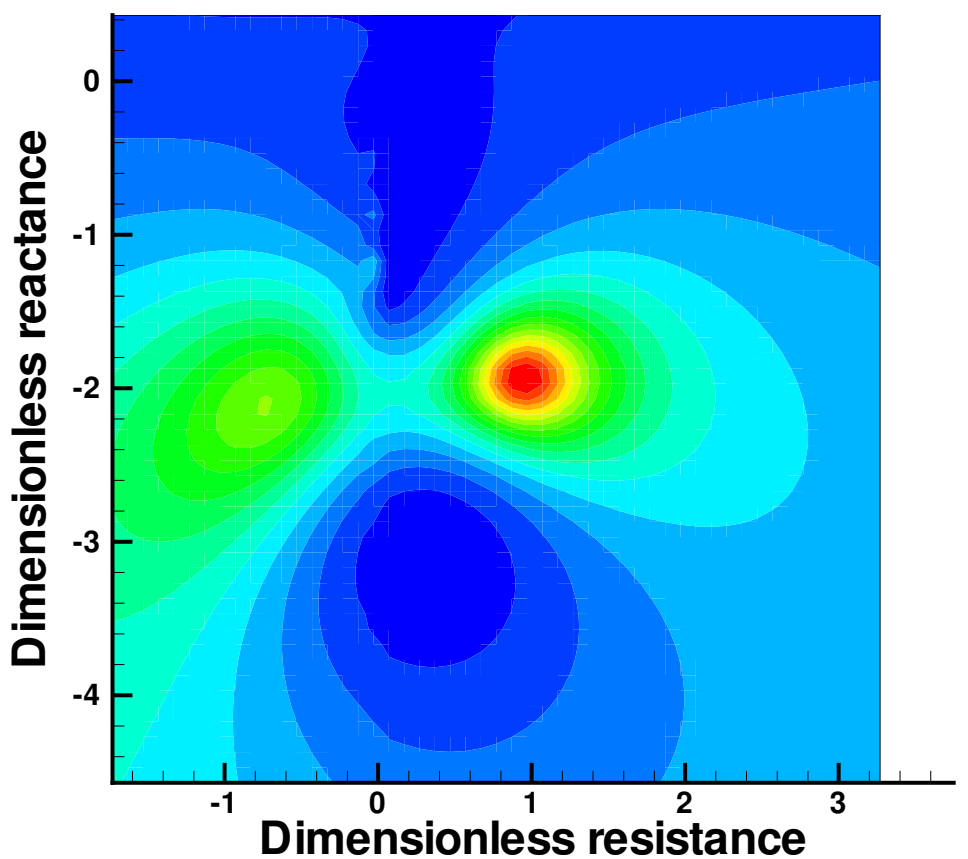

Figure 7. Contour plot of objective function for the conventional liner at Mach 0.3 and $0.4 \mathrm{kHz}$. 


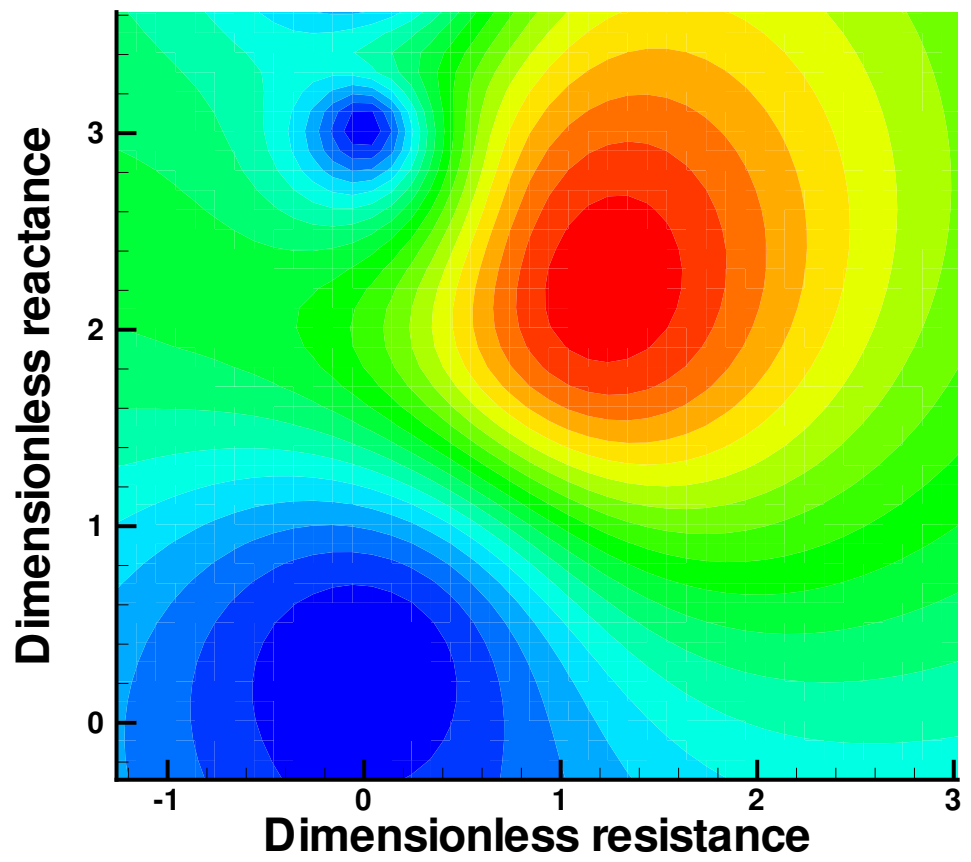

Figure 8. Contour plot of objective function for the conventional liner at Mach 0.0 and $3.0 \mathrm{kHz}$. 